\title{
High Glucose Represses hERG K+ Channel Expression through Trafficking Inhibition
}

\author{
Yuan-Qi Shia Meng Yana Li-Rong Liua Xiao Zhanga Xue Wanga \\ Huai-Ze Genga Xin Zhao ${ }^{a}$ Bao-Xin Lia,b \\ aDepartment of Pharmacology, Harbin Medical University, Harbin, bState-Province Key Laboratory of \\ Biopharmaceutical Engineering, Harbin, China
}

\section{Key Words}

Diabetes mellitus $•$ High-glucose $\cdot$ hERG $\bullet$ LQT $・$ Unfolded protein response $・$ Insulin

\begin{abstract}
Background/Aims: Abnormal QT prolongation is the most prominent cardiac electrical disturbance in patients with diabetes mellitus (DM). It is well known that the human ether-ago-go-related gene ( $\mathrm{hERG}$ ) controls the rapid delayed rectifier $\mathrm{K}^{+}$current $\left(\mathrm{I}_{\mathrm{Kr}}\right)$ in cardiac cells. The expression of the hERG channel is severely down-regulated in diabetic hearts, and this down-regulation is a critical contributor to the slowing of repolarization and QT prolongation. However, the intracellular mechanisms underlying the diabetes-induced hERG deficiency remain unknown. Methods: The expression of the hERG channel was assessed via western blot analysis, and the hERG current was detected with a patch-clamp technique. Results: The results of our study revealed that the expression of the hERG protein and the hERG current were substantially decreased in high-glucose-treated hERG-HEK cells. Moreover, we demonstrated that the high-glucose-mediated damage to the hERG channel depended on the down-regulation of protein levels but not the alteration of channel kinetics. These discoveries indicated that high glucose likely disrupted hERG channel trafficking. From the western blot and immunoprecipitation analyses, we found that high glucose induced trafficking inhibition through an effect on the expression of Hsp90 and its interaction with hERG. Furthermore, the high-glucose-induced inhibition of hERG channel trafficking could activate the unfolded protein response (UPR) by up-regulating the expression levels of activating transcription factor- 6 (ATF-6) and the ER chaperone protein calnexin. In addition, we demonstrated that 100 $\mathrm{nM}$ insulin up-regulated the expression of the hERG channel and rescued the hERG channel repression caused by high glucose. Conclusion: The results of our study provide the first evidence of a high-glucose-induced hERG channel deficiency resulting from the inhibition of channel trafficking. Furthermore, insulin promotes the expression of the hERG channel and ameliorates the high-glucose-induced inhibition of the hERG channel.
\end{abstract}

Copyright (C) 2015 S. Karger AG, Basel

Bao-Xin Li

KARGER 125
Department of Pharmacology, Harbin Medical University, No.157 Baojian Road, Harbin Heilongjiang Province, 150081 (P. R. China)

Tel. +86 451 86671354, Fax +86 451 86667511, E-Mail libx64@hotmail.com 


\begin{tabular}{|c|c|c|}
\hline Cellular Physiology & Cell Physiol Biochem 2015;37:284-296 & \\
\hline and Biochemistry & $\begin{array}{l}\text { DOI: 10.1159/000430353 } \\
\text { Published online: August 24, } 2015\end{array}$ & $\begin{array}{l}\text { O } 2015 \text { S. Karger AG, Basel } \\
\text { www.karger.com/cpb }\end{array}$ \\
\hline
\end{tabular}

\section{Introduction}

An abnormal prolongation of the QT interval is a prominent electrical disorder in patients with diabetes mellitus (DM). The prevalence is up to approximately $25 \%$ in both type 1 insulin-dependent DM (IDDM) and type 2 insulin-independent DM (NIDDM) patients. QT prolongation has been proposed as a predictor of mortality in IDDM and NIDDM because this abnormality is associated with an increased risk of sudden cardiac death subsequent to lethal ventricular arrhythmias [1-4]. The QT interval reflects the integrated action potential duration (APD) in cardiac myocytes. Ion currents play an important role in cardiac APD, and multiple ion currents are decreased in the experimental animal models of diabetes [5, 6]. Therefore, sufficient knowledge of the ionic mechanisms underlying the diabetic QT in diabetes is urgently needed.

Long QT syndrome (LQTs) can be induced by a reduction in the rapid delayed rectifier potassium current $\left(\mathrm{I}_{\mathrm{Kr}}\right)$, the pore-forming subunits for which are encoded by the human ether-a-go-go related gene (hERG) $[7,8]$. It has been reported that the expression of the hERG channel is significantly down-regulated in diabetic hearts, and this down-regulation is a critical contributor to diabetic repolarization slowing and QT prolongation [6, 9]. However, there is still an incomplete understanding of the diabetes-induced $\mathrm{I}_{\mathrm{Kr}} / \mathrm{hERG}$ dysfunction. Immature hERG channels are folded and initially glycosylated in the ER. Subsequently, the incompletely glycosylated channels are sent to the Golgi to be fully glycosylated, followed by translocation to the cell membrane. The phosphorylation of hERG potassium channel is mainly achieved by protein kinase A, B and C (PKA, PKB, PKC) [10, 11]. The hERG channel trafficking process is monitored through quality control $(\mathrm{QC})$ and the unfolded protein response (UPR), comprising a complex molecular chaperone system [12]. Therefore, hERG protein trafficking is a major factor in hERG channel expression and is under the regulation of a cytosolic chaperone network [13]. Numerous studies have shown that the inhibition of hERG protein trafficking could reduce hERG channel expression. However, it remains uncertain whether hERG protein trafficking is altered in diabetic cardiomyocytes. Therefore, the aim of our study was to further the current mechanistic understanding of diabetesinduced hERG channel abnormalities, which may aid in developing potential therapeutic approaches for this disease.

In our study, we incubated cells with high-glucose medium to generate an in vitro cellular model $[14,15]$. We investigated whether the hERG protein and current were inhibited by high glucose levels in HEK293 cells that stably expressed the wild-type hERG gene. We also focused on whether high-glucose incubation could induce a trafficking dysfunction and subsequently increase degradation. Finally, we determined whether insulin affected the hERG channel expression that had been reduced by high glucose.

\section{Materials and Methods}

\section{Cell culture}

HEK293 cells stably expressing the wild-type hERG gene were used to study the effects of different concentrations of high glucose on the hERG channel. The hERG-HEK cells were cultured at $37^{\circ} \mathrm{C}$ in a humidified atmosphere of $5 \% \mathrm{CO}_{2}$ in Dulbecco's modified Eagle's Medium (DMEM; HyClone, Logan, UT, USA) supplemented with $10 \%$ (v/v) foetal bovine serum (FBS; TBD, Tianjin, China). For the hERG-HEK cells, the medium was supplemented with $400 \mathrm{mg} / \mathrm{ml}$ of geneticin (G-418, Invitrogen, Carlsbad, CA, USA) for the selection of stably transfected cells. For electrophysiological studies, the cells were harvested via trypsinization, washed twice with standard DMEM, and stored in the same medium at room temperature until further use. The cells were studied within $8 \mathrm{~h}$ of harvesting.

\section{Western blot analysis}

The expression of high levels of the hERG protein was monitored using western blot experiments. High glucose at different concentrations was diluted and added to hERG-HEK cells for $48 \mathrm{~h}$ at $37^{\circ} \mathrm{C}$ prior 


\section{Cellular Physiology Cell Physiol Biochem 2015;37:284-296 \begin{tabular}{l|l} 
and Biochemistry Published online: August 24, 2015 & $\begin{array}{l}\text { C) 2015 S. Karger AG, Basel } \\
\text { www.karger.com/cpb }\end{array}$ \\
\hline
\end{tabular} \\ Shi et al.: High Glucose Inhibits hERG Trafficking}

to the western blot analysis. The cells were placed on ice and washed 3 times with $3 \mathrm{ml}$ of ice-cold PBS. Subsequently, $60 \mu$ l of RIPA buffer (Bi Yun Tian, Jiangsu, China) and $0.6 \mu$ l of PMSF (Shenneng Bocai, Shanghai, China) were added to the plates, and the cells were scraped from the plates and transferred into tubes. Proteins ( $150 \mu \mathrm{g}$ per sample) were separated using SDS-PAGE, transferred onto nitrocellulose membranes (Stratagene, La Jolla, CA) and incubated with primary antibodies against GAPDH (Affinity Reagents) and a specific polyclonal rabbit anti-hERG antibody (Santa Cruz Biotechnology, CA) at a 1:100 dilution. Goat anti-rabbit Alexa Fluor 700 (dilution 1:2,000, Molecular Probes, Eugene, OR) was used as the secondary antibody. An Odyssey infrared fluorescent scanning system (LI-COR, Lincoln, NE) was used to detect the membrane proteins. The band densities were quantified through densitometry using the Scion Image software (Scion, Frederick, MD). The data were normalized to GAPDH.

\section{Immunoprecipitation}

The hERG-HEK cells were washed 3 times with PBS on ice and transferred to a 1.5-ml tube. The cells were centrifuged for $5 \mathrm{~min}$ at 3,000 rpm, and $300 \mu \mathrm{l}$ of RIPA plus $3 \mu \mathrm{l}$ of PMSF was added to the pellet. The uniformly mixed suspension was placed on ice for $10 \mathrm{~min}$ and then centrifuged for $10 \mathrm{~min}$ at 12,000 $\mathrm{rpm}$. The supernatant protein concentration was measured using the Bradford method. Two micrograms of anti-hERG, anti-Hsp70 or anti-Hsp90 antibody was separately mixed with the protein, and the mixture was placed on a $360^{\circ}$ shaker overnight at $4^{\circ} \mathrm{C}$. Subsequently, $40 \mu \mathrm{l}$ of beads (Santa Cruz A-G SC-2003) was added to the mixture, and the tubes were placed on a shaker overnight at $4^{\circ} \mathrm{C}$. The mixture was centrifuged at 1,500 rpm for $5 \mathrm{~min}$, and the pellet was washed 6 times with TBST on ice. Next, $100 \mu \mathrm{l}$ of loading buffer was added to the pellet, and the tubes were boiled for $10 \mathrm{~min}$, followed by centrifugation. The supernatants were collected and separated via SDS-PAGE; proteins were transferred to a PVDF membrane, which was then blocked with 5\% non-fat milk, incubated with anti-hERG, anti-Hsp90, or anti-Hsp70 (Santa Cruz, sc8-17) antibody, and then a secondary antibody. The supernatants were collected and analysed through western blot analysis.

\section{Patch-clamp recording techniques}

For the electrophysiological recordings, submaximally confluent cells were used after 1-3 days of culture at a seeding density of $1-4 \times 10^{5}$ cells per flask (NEST). After incubation with high glucose for 48 $\mathrm{h}$, the hERG-HEK cells were trypsinized, centrifuged and stored at $4^{\circ} \mathrm{C}$ in a bath solution for $1 \mathrm{~h}$ to stabilize separated single cells. The cells were transferred to a small cell bath mounted on the stage of an inverted microscope (IX-70; Olympus, Tokyo) and incubated for approximately $10 \mathrm{~min}$ to facilitate attachment to the glass bottom. The cells were then continuously superfused at a rate of $1.5 \mathrm{ml} / \mathrm{min}$.

The hERG currents were measured using the whole-cell configuration patch-clamp recording technique. The whole-cell configuration was formed using a glass pipette with a tip resistance of 2-5 $\mathrm{mV}$ when filled with the following pipette solution: $130 \mathrm{mM} \mathrm{KCl}, 1 \mathrm{mM} \mathrm{MgCl}{ }_{2} \bullet 6 \mathrm{H}_{2} \mathrm{O}, 10 \mathrm{mM}$ HEPES, $5 \mathrm{mM} \mathrm{Mg}$ ATP, $5 \mathrm{mM}$ EGTA, and $0.1 \mathrm{mM}$ GTP (pH 7.3 with $\mathrm{KOH}$ ). The extracellular solution contained $136 \mathrm{mM} \mathrm{NaCl}, 5.4$ $\mathrm{mM} \mathrm{KCl}, 5 \mathrm{mM}$ HEPES, $1 \mathrm{mM} \mathrm{MgCl} \bullet_{2} \cdot 6 \mathrm{H}_{2} \mathrm{O}, 1 \mathrm{mM} \mathrm{CaCl}_{2}$ and $10 \mathrm{mM}$ glucose (pH 7.4 with $\mathrm{NaOH}$ ). An Axopatch200B patch-clamp amplifier was used to record the membrane current. Computer software (Clampex 9.2; Axon Instruments, USA) was used to generate the voltage clamp protocols and acquire data. The capacitance and series resistance compensation were optimized. The data were recorded on a computer via Digidata 1322A and analysed using Clampfit 9.2 (Axon Instruments) and Prism (GraphPad Inc., San Diego, CA, USA) software. Graphical fits of the data were generated using previously described standard equations [16].

\section{Statistical analyses}

The data are presented as the means \pm S.E.M. Statistic analysis was performed using the SPSS 16.0 software to evaluate statistically significant differences. Student's t-test was used for comparisons between two groups, and a one-way or two-way analysis of variance (ANOVA) was used to analyse significant differences between groups under different conditions. Differences between the two groups were considered significant when the P-values were $<0.05$ (two-tailed). All the graphs were drawn using GraphPad Prism 5.0 software. 


\section{Results}

High-glucose treatment reduces $h E R G$ protein levels and hERG current

High blood glucose is a major factor for cardiomyopathy in patients with diabetes DM [17]. Therefore, in the present study, we incubated cells with 50, 75 and $100 \mathrm{mM}$ glucose to mimic hyperglycaemia (HG). To investigate the long-term effects of high glucose on the expression of hERG channels, western blot analysis was performed. As shown in Fig. 1A, hERG protein levels were significantly decreased after incubation with different highglucose concentrations for 48 h. Fig. $1 \mathrm{~A}$ indicates the densities of the hERG 155- and 135$\mathrm{kDa}$ bands after high-glucose treatment relative to the respective control values. Highglucose treatment reduced the density of the $155-\mathrm{kDa}$ band by $25.57 \% \pm 2.32 \%$ (50 $\mathrm{mM})$,

\section{A}
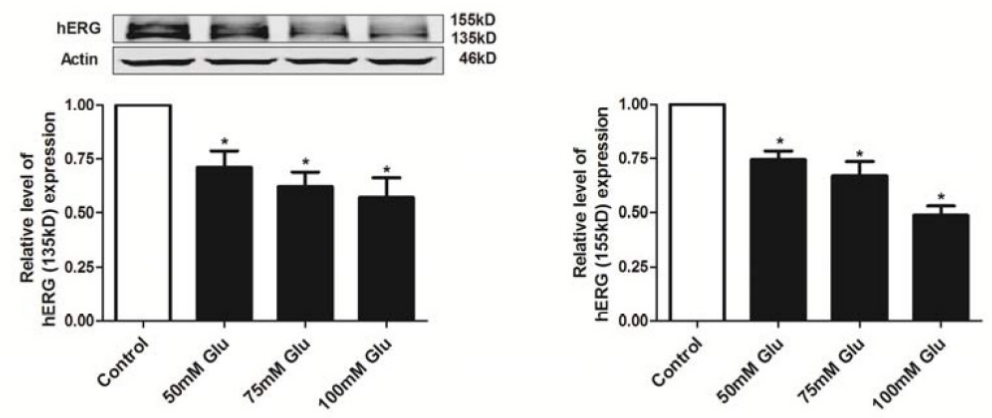

B
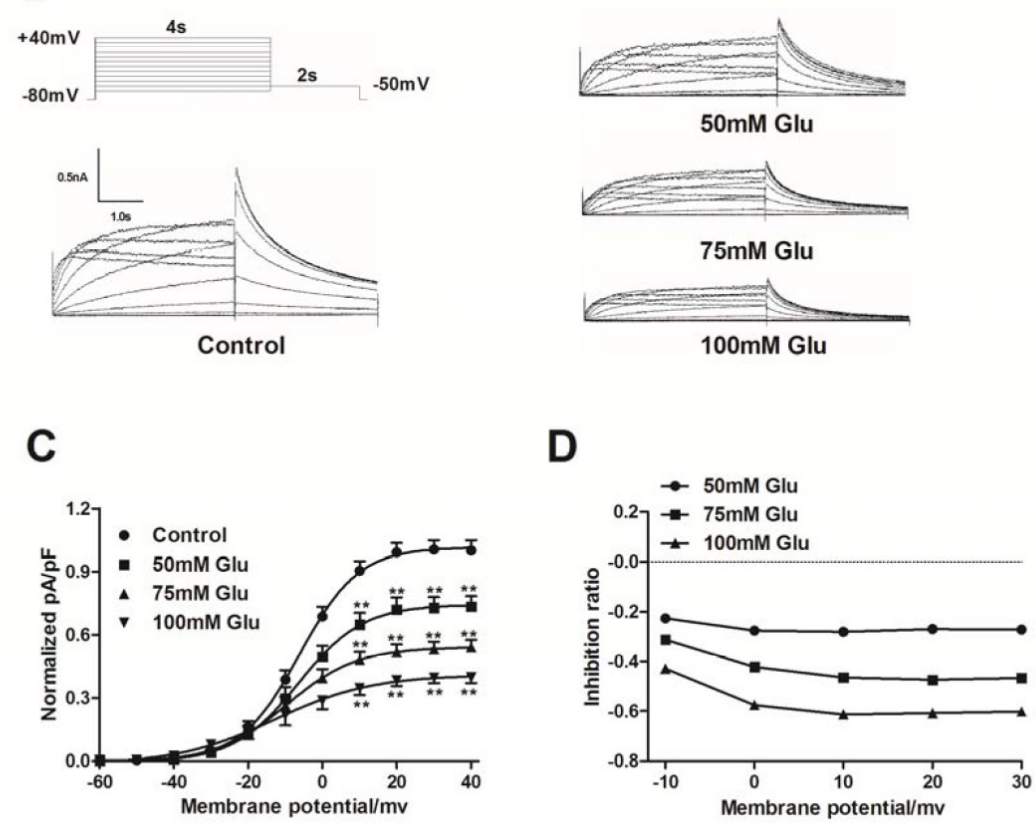

Fig. 1. The different high-glucose concentrations down-regulated both the mature and immature forms of the hERG channel. (A) Western blot results showing that high-glucose significantly reduced both the 135$\mathrm{kDa}$ and 155-kDa of hERG channel in a concentration-dependent manner (50, 75 and $100 \mathrm{mM})$. ${ }^{*} \mathrm{P}<0.05$ vs. control, $n=6$. (B) Voltage clamp protocol and representative hERG current traces recorded from different experimental groups under the same conditions as described above. (C) Normalized I-V relationships for the tail current in the absence and presence of high glucose. High glucose significantly reduced the hERG current. ${ }^{*} \mathrm{P}<0.01$ vs. control, $\mathrm{n}=10$. (D) The inhibition ratio of the high-glucose voltage dependence peak tail current calculated using the function $\left(\mathrm{I}_{\text {High-glucose }}-\mathrm{I}_{\text {Control }}\right) / \mathrm{I}_{\text {Control }}{ }^{*}$ 
A

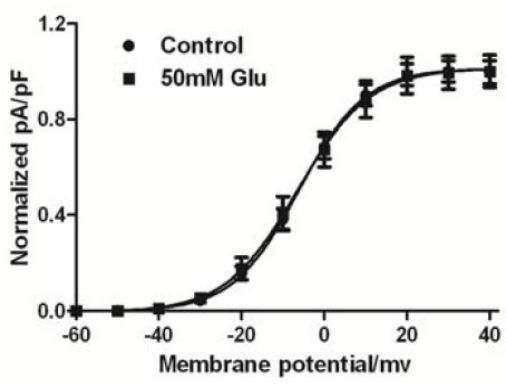

C

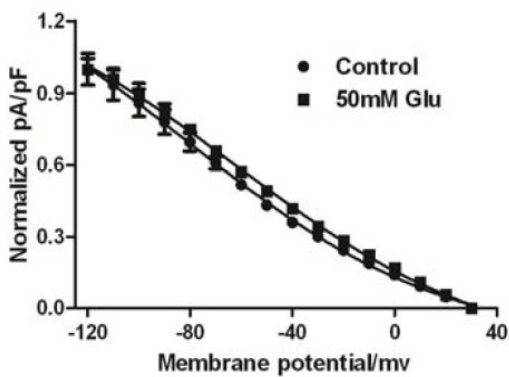

E

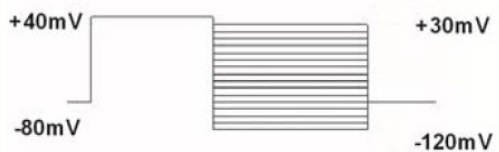

B
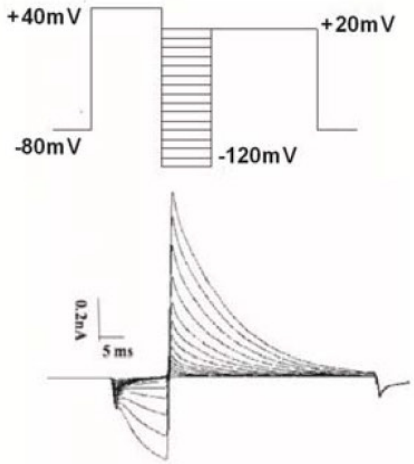

D
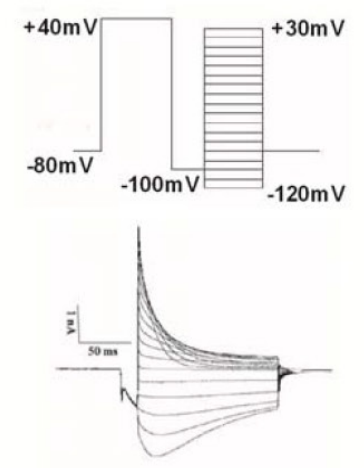

$\mathbf{F}$

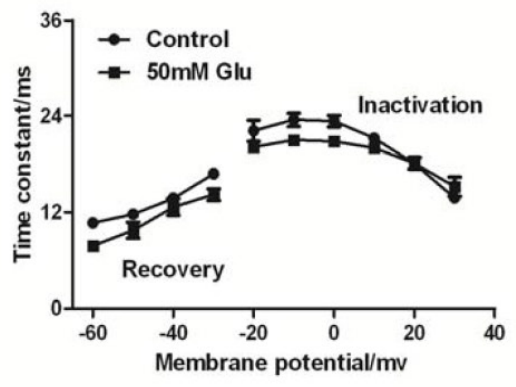

Fig. 2. The effect of high glucose on hERG channel kinetics. (A) Voltage-dependent activation curves for the control group and following exposure to high glucose for $48 \mathrm{~h}$. (B) Voltage clamp protocol and representative current tracing for steady-state inactivation. (C) The effect of high glucose on the inactivation curve after incubation for 48 h. (D) Voltage clamp protocol and representative current tracing for the onset of inactivation. (E) Voltage clamp protocol and representative current tracing for the recovery from inactivation. (F) The effect of high glucose on the time constant for the onset of inactivation and recovery from inactivation after incubation for $48 \mathrm{~h}(\mathrm{n}=10)$.

$33.05 \% \pm 5.24 \%(75 \mathrm{mM})$ and $51.35 \% \pm 3.46 \%(100 \mathrm{mM})$. The density of the 135-kDa band was reduced by $28.99 \% \pm 4.64 \%(50 \mathrm{mM}), 37.83 \% \pm 3.83 \%(75 \mathrm{mM})$ and $42.89 \% \pm 5.28 \%$ $(100 \mathrm{mM})$.

To determine whether the reduction in the level of mature hERG protein causes a dysfunction in the hERG current, patch-clamp recordings were used to detect the hERG 
A

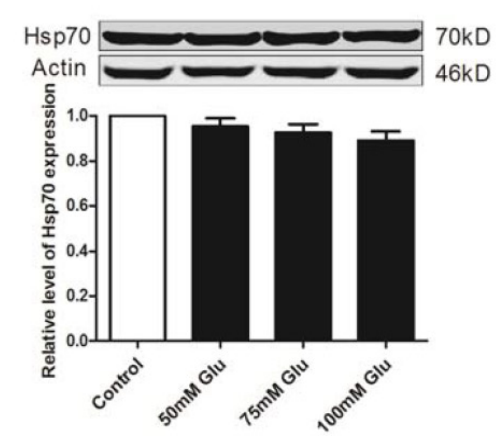

C
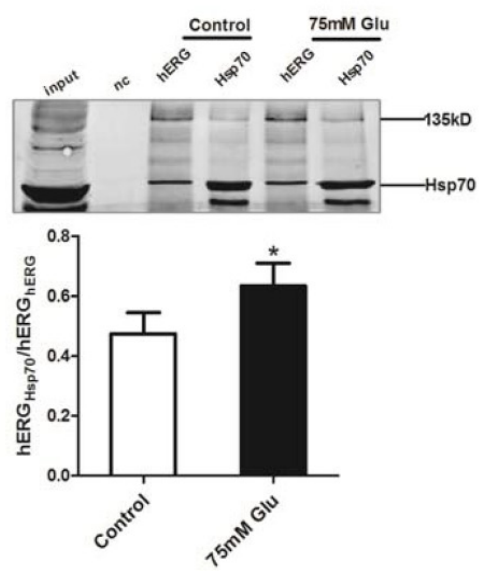

B
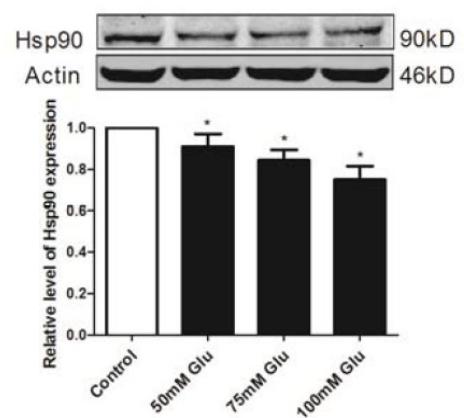

D
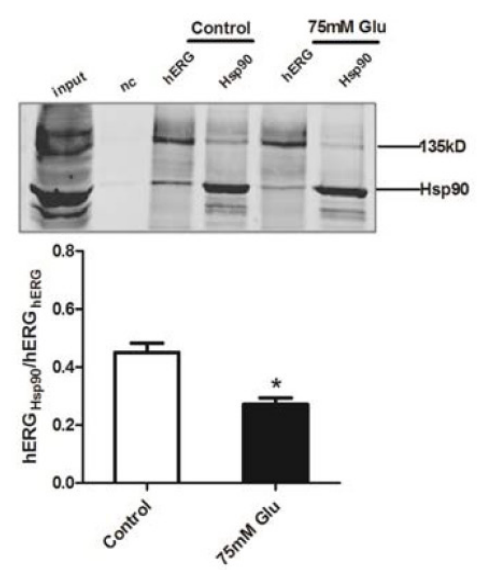

Fig. 3. High-glucose incubation disrupts the interaction between hERG and heat shock proteins. (A-B) Western blot results for Hsp90 and Hsp70 expression in the presence of high glucose for $48 \mathrm{~h}$. Hsp90 was decreased in a concentration-dependent manner, while Hsp70 was unchanged. ${ }^{*} \mathrm{P}<0.05$ vs. control, $\mathrm{n}=4$. (C-D) Immunoprecipitation results revealing the interactions of hERG with Hsp70 or Hsp90 after treatment with $75 \mathrm{mM}$ glucose. The hERG protein interaction with Hsp90 was decreased, while the hERG protein interaction with Hsp70 was increased. $* \mathrm{P}<0.05$ vs. control, $\mathrm{n}=3$.

current. Fig. 1B and C illustrates the effects of high glucose on the tail current of the hERG channel. The hERG current was substantially reduced in a concentration-dependent manner with high-glucose treatment. The ratio of inhibition by high glucose was calculated using the tail current from -20 to $30 \mathrm{mV}$ and the function $\left(\mathrm{I}_{\text {high-glucose }}-\mathrm{I}_{\text {Control }}\right) / \mathrm{I}_{\text {control }}$. As depicted in Fig. $1 \mathrm{E}$, the ratios in the $50 \mathrm{mM}, 75 \mathrm{mM}$ and $100 \mathrm{mM}$ groups were $-0.27,-0.47$ and -0.60 at +30 $\mathrm{mV}$, respectively.

\section{Long-term treatment with high glucose does not affect channel kinetics}

We used patch-clamp recordings to detect the long-term effects of high glucose on the hERG channel kinetics. The steady-state activation curves in Fig. 2A were constructed after normalizing the tail currents at various test potentials to the maximum current and were plotted against the pre-pulse voltages. The data were fitted using the Boltzmann function. As shown in Fig. $2 \mathrm{~A}$, the activation voltage $\left(\mathrm{V}_{1 / 2}\right)$ values were $-6.12 \pm 0.57 \mathrm{mV}$ in the control group and $-6.44 \pm 0.64 \mathrm{mV}$ in the $50 \mathrm{mM}$ high-glucose group. The corresponding slope factor $(k)$ values were $7.68 \pm 0.51$ and $8.67 \pm 0.58$, respectively. High glucose did not affect the activation of the hERG channel.

Furthermore, we determined whether high glucose affects the inactivation of the hERG channel. Fig. 2B illustrates the voltage clamp protocol and representative current tracing for 
A
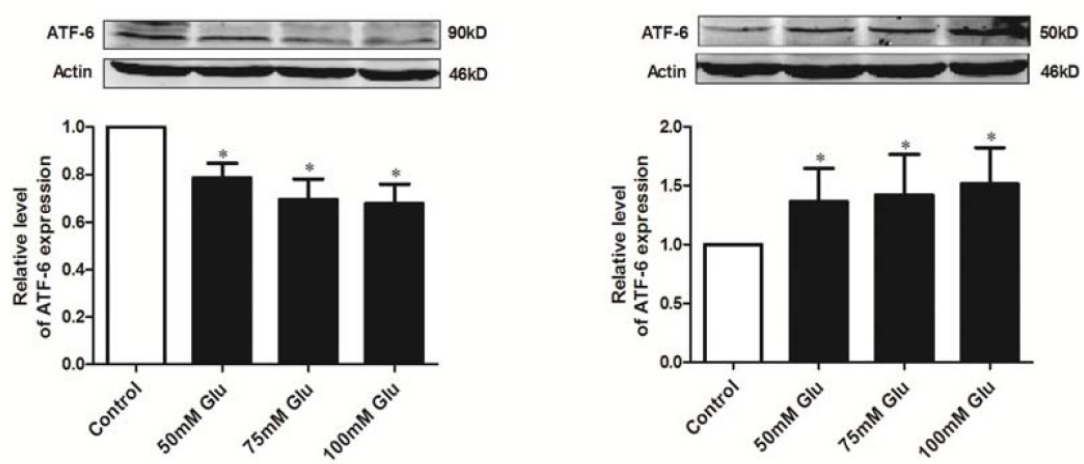

B
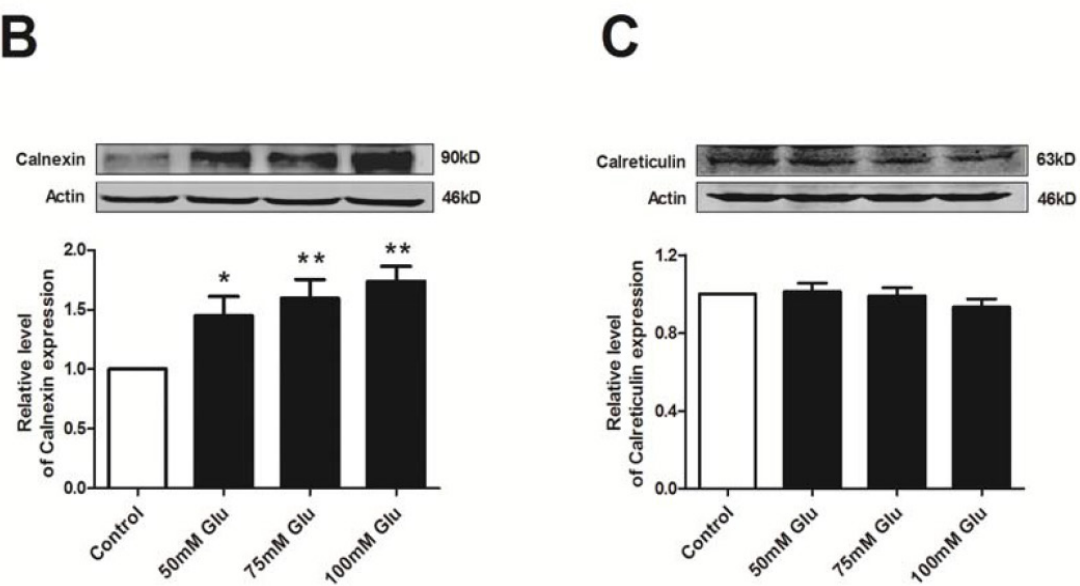

Fig. 4. The unfolded protein response is activated after incubating hERG-HEK cells with high glucose for 48 h. (A) Expression of ATF-6 ( 90 kDa) and cleaved ATF-6 ( $>50 \mathrm{kDa})$ after high-glucose incubation for $48 \mathrm{~h}$. High-glucose incubation remarkably reduced the $90-\mathrm{kDa}$ form of ATF- 6 and as a result, up-regulated the 50-kDa form of ATF-6. (B-C) Western blots results and statistics showing the expression of calnexin and calreticulin. High-glucose incubation increased the expression of calnexin but not calreticulin. ${ }^{*} \mathrm{P}<0.05$, ${ }^{* *} \mathrm{P}<0.01$ vs. control, $\mathrm{n}=4$.

a steady-state inactivation. Fig. $2 \mathrm{C}$ demonstrates the effect of high glucose on the inactivation curve after a 48-h incubation. High glucose did not significantly shift the inactivation curve: the $\mathrm{V}_{1 / 2}$ was $-79.58 \pm 9.87 \mathrm{mV}$ in the control group and $-69.82 \pm 7.81 \mathrm{mV}$ in the $50 \mathrm{mM}$ highglucose group, and the $k$ was $-50.27 \pm 9.25$ and $-50.73 \pm 9.12$, respectively.

The time courses for the development of and recovery from inactivation were also assessed. Fig. 2D and E depicts the voltage clamp protocol and a representative current tracing for the onset of inactivation and recovery from inactivation. Fig. 2F shows the differences in the time constants for steady-state inactivation and recovery between the control group and the group exposed to $50 \mathrm{mM}$ high glucose were not significant. Moreover, we observed that high glucose inhibited the hERG current but did not alter the kinetics of channel gating, suggesting that the high-glucose-mediated damage of the hERG channel is dependent upon a reduction in the protein level.

\section{High-glucose induces a trafficking deficiency of the hERG channel}

The inhibition of trafficking is known to be one mechanism for the hERG deficiency. Because the cytosolic chaperones Hsp70 and Hsp90 participate in hERG trafficking, we used western blot analysis to investigate whether high glucose alters the steady-state expression 


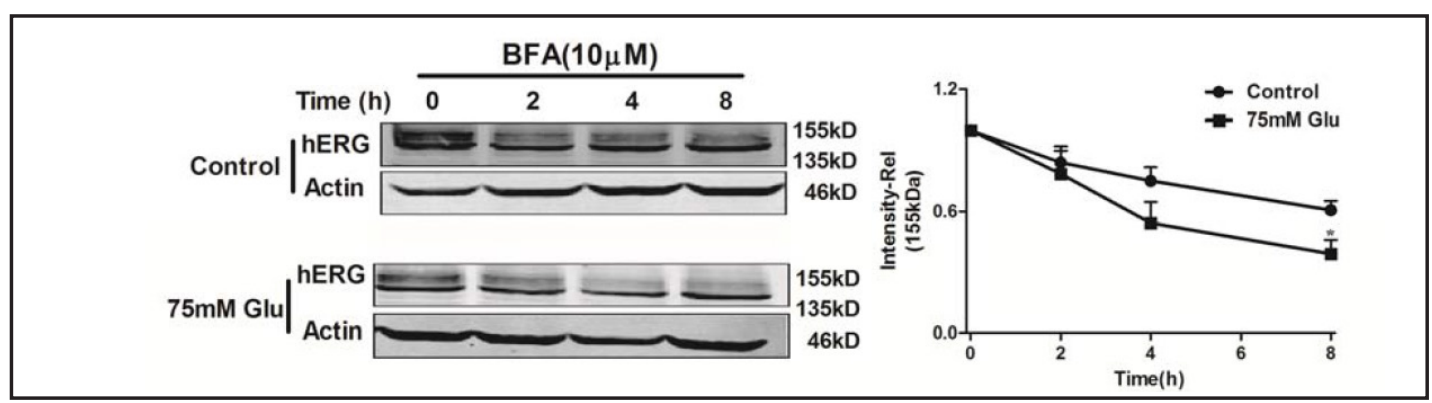

Fig. 5. Western blot results reveal that high-glucose incubation accelerated the degradation of the mature form of the hERG channel. hERG-HEK cells were treated without (control) or with high glucose (75 mM) in the presence of BFA $(10 \mu \mathrm{M})$, which blocks hERG protein transport from the ER to the Golgi. Experimental cells were harvested at the indicated time points for western blot analysis. The intensities of the 155-kDa mature hERG bands at each time point were normalized to the time 0 h value. ${ }^{*} \mathrm{P}<0.05$ vs. control, $\mathrm{n}=3$.

level of endogenous Hsp90 and/or Hsp70. Fig. 3A and B shows that the expression of Hsp90 is decreased in the presence of high glucose, whereas Hsp70 expression is not changed. In addition, we used immunoprecipitation to detect whether high glucose modifies the association of hERG channels with Hsp70 and/or Hsp90. As indicted by Fig. 3C and D, in the presence of high glucose at $75 \mathrm{mM}$, the interaction between the hERG protein and Hsp90 was significantly decreased, while the interaction between the hERG protein and Hsp70 was increased. Taken together, these results demonstrated that the high-glucose-induced trafficking deficiency of hERG channels likely reflects alterations in the interactions between the hERG proteins and chaperones.

\section{High glucose activates unfolded protein response (UPR)}

The UPR is a process that prevents the accumulation of unfolded proteins in the ER. Active transcription factor-6 (ATF-6) has been identified as a marker protein of the UPR; therefore, we further detected the effect of high glucose on ATF-6. Fig. 4A reveals that the expression of ATF-6 ( $\sim 90 \mathrm{kDa})$ was significantly decreased, while the expression of the cleaved form of ATF-6 ( $\sim 50 \mathrm{kDa})$ was proportionally increased. These results demonstrate that ER stress is activated after incubation with high glucose for $48 \mathrm{~h}$. Calnexin and calreticulin, identified as chaperone proteins and downstream targets of cleaved ATF-6, have been reported to interact with unfolded hERG proteins and subsequently contribute to their degradation. Based on these findings, we further tested whether calnexin/calreticulin expression was altered by high glucose treatment. Based upon the results depicted in Fig. 4B and C, we found that the expression of calnexin was significantly increased, while the expression of calreticulin was not altered in the presence of high glucose for $48 \mathrm{~h}$.

\section{High-glucose enhances the degradation of hERG channels}

To assess the effects of high glucose on the degradation of mature hERG channels, we used the Golgi transit inhibitor brefeldin A (BFA) to inhibit the conversion of hERG from the immature 135-kDa form to the mature 155-kDa form [18]. The hERG-HEK cells were pretreated with BFA $(10 \mu \mathrm{M})$ for $1 \mathrm{~h}$ and then cultured in the absence or presence of high glucose at $75 \mathrm{mM}$ in the continued presence of BFA. Western blot analysis was used to detect the expression of the $155-\mathrm{kDa}$ band at different time points after BFA treatment. The degradation rate of hERG was indicated by the normalized 155-kDa band intensity. As shown in Fig. 5, the expression of mature hERG protein is reduced in a time-dependent manner in presence of BFA. The image on the right side of Fig. 5 indicates that, in control cells, incubation with BFA for $8 \mathrm{~h}$ decreased the 155-kDa hERG band intensity by approximately $40 \%$. In contrast, in the high-glucose-treated cells, the 155-kDa hERG band intensity decreased approximately $62 \%$. Therefore, these results demonstrated that the degradation rate of mature hERG channels was significantly enhanced after exposure to high glucose at $75 \mathrm{mM}$. 
A
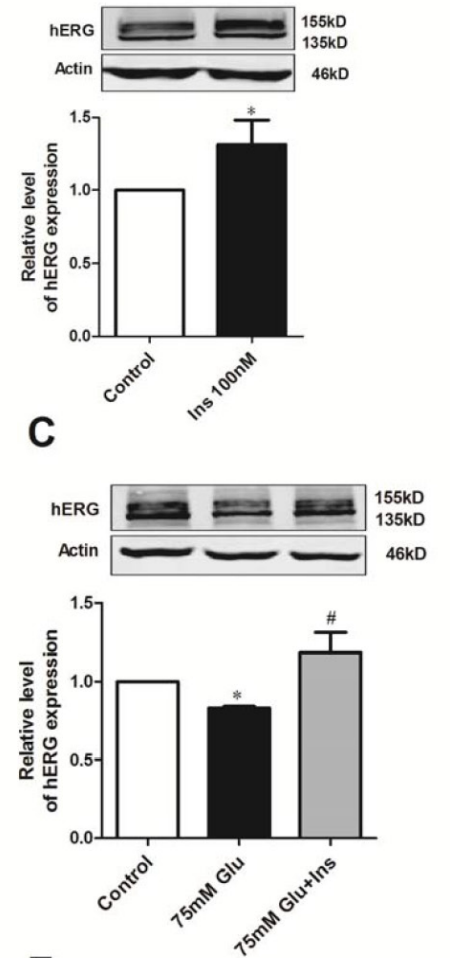

E

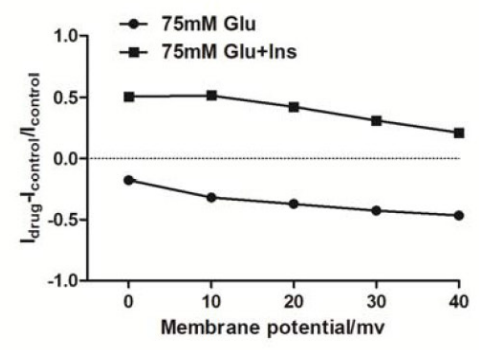

B

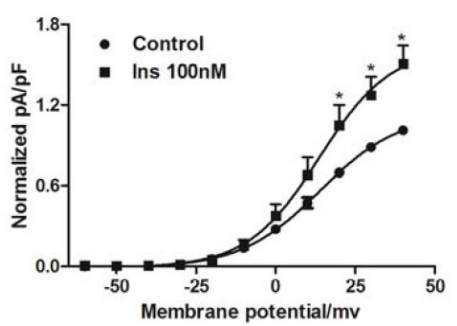

D

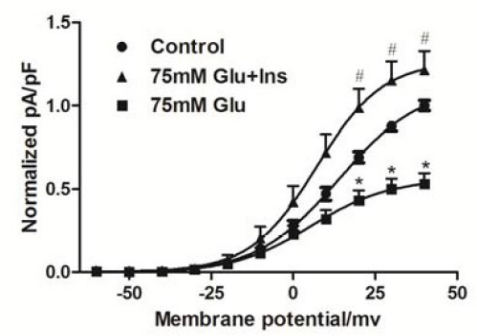

F

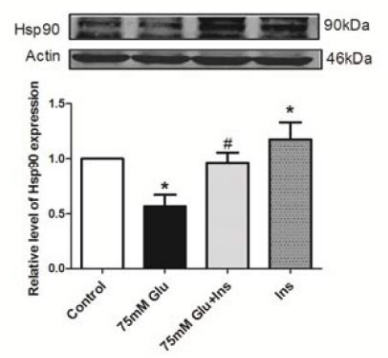

Fig. 6. Insulin reversed the reduction of the 155-kDa hERG expression and hERG current induced by high glucose. (A) The up-regulation of hERG channel expression after treatment with high glucose for $48 \mathrm{~h}$, determined via western blotting. ${ }^{*} \mathrm{P}<0.05$ vs. control, $\mathrm{n}=4$. (B) Normalized I-V relationships for the hERG tail current. Insulin increases the hERG tail current. ${ }^{*} \mathrm{P}<0.05$ vs. control, $\mathrm{n}=10$. (C) The decreased expression of the hERG protein caused by high glucose is up-regulated after exposure to $100 \mathrm{nM}$ insulin. $* \mathrm{P}<0.05$ vs. control, $\# \mathrm{P}<0.05$ vs. high glucose, $\mathrm{n}=4$. (D) Normalized I-V relationships for the tail current before and after 75 $\mathrm{mM}$ high-glucose treatment are restored with $100 \mathrm{nM}$ insulin. ${ }^{*} \mathrm{P}<0.05$ vs. control, $\mathrm{n}=10$. (E) The efficiency of high glucose and the insulin voltage dependence peak tail current were calculated using the functions $\left(\mathrm{I}_{\text {High-glucose }}-\mathrm{I}_{\text {Control }}\right) / \mathrm{I}_{\text {Control }}$ and $\left(\mathrm{I}_{\text {Insulin }}-\mathrm{I}_{\text {Control }}\right) / \mathrm{I}_{\text {Control }}$, respectively. (F) Western blotting results revealing Hsp90 expression in the presence high glucose and insulin for $48 \mathrm{~h}$. The depression of Hsp90 expression caused by high glucose is recovered after co-incubation with insulin for $48 \mathrm{~h}$. Insulin increased the expression of Hsp90. $* \mathrm{P}<0.05$ vs. control, $\# \mathrm{P}<0.05$ vs. high glucose, $\mathrm{n}=4$.

\section{Insulin rescues $h E R G$ deficiency after high-glucose treatment}

We determined the effect on hERG channel expression of long-term treatment (48 h) with $100 \mathrm{nM}$ insulin by assessing the levels of mature hERG protein and hERG current. As illustrated in Fig. 6A, the results indicated that the expression of the 155-kDa hERG band 
increased after treatment with $100 \mathrm{nM}$ insulin for $48 \mathrm{~h}$. Subsequently, we used patch-clamp recordings to detect the effect of the chronic application of insulin on the hERG current. The increased expression of the mature hERG protein on western blots was also reflected in the electrophysiological recordings. The results shown in Fig. 6B revealed that the hERG tail current is significantly increased after treatment with $100 \mathrm{nM}$ insulin for $48 \mathrm{~h}$.

We also used western blot analysis to investigate whether $100 \mathrm{nM}$ insulin could rescue the hERG protein reduction caused by high glucose at $75 \mathrm{mM}$. As depicted in Fig. 6C, the expression level of the hERG protein was significantly reduced after incubation with $75 \mathrm{mM}$ glucose. However, the application of $100 \mathrm{nM}$ insulin ameliorated the inhibition of the hERG channel caused by high glucose. To further demonstrate whether the hERG protein that was restored by insulin treatment is functional, we used a patch-clamp technique to record the hERG current under the same conditions as described above. In a similar manner, $100 \mathrm{nM}$ insulin reversed the down-regulation of the hERG current induced by high glucose at $75 \mathrm{mM}$ (Fig. 6D). The inhibitory ratio of high glucose was calculated from the tail current at $+30 \mathrm{mV}$ using the function $\left(\mathrm{I}_{\text {high-glucose }}-\mathrm{I}_{\text {Control }}\right) / \mathrm{I}_{\text {Control }}$ As indicated in Fig. $6 \mathrm{E}$, the ratio was -0.44 at 30 $\mathrm{mV}$. The rescue ratio for $100 \mathrm{nM}$ insulin was 0.31 at $+30 \mathrm{mV}$, calculated using the function $\left(\mathrm{I}_{\text {Insulin }}-\mathrm{I}_{\text {Control }}\right) / \mathrm{I}_{\text {Control }}$.

To determine how insulin reverses the down-regulation of the hERG channel, we examined the effect of insulin on Hsp90 protein expression. As displayed in Fig. 6F, the expression of Hsp90 was decreased in the presence of high glucose, and insulin significantly recovered the Hsp90 expression. Moreover, the expression of Hsp90 increased after incubation with $100 \mathrm{nM}$ insulin. These data indicated that insulin likely ameliorated hERG inhibition through improved hERG protein trafficking.

\section{Discussion}

In the present study, we demonstrated for the first time that both hERG protein expression and hERG current were decreased in high-glucose-treated hERG-HEK cells. A novel mechanism was revealed, which is that high glucose induces a hERG channel trafficking deficiency by inhibiting the expression of Hsp90 and the hERG/Hsp90 complex. Subsequently, the ER stress response and increased protein degradation are activated in high-glucose-treated hERG-HEK cells. Moreover, the application of $100 \mathrm{nM}$ insulin restored the expression of the hERG protein and hERG current. These findings indicate that the inhibition of trafficking represents a potential mechanism underlying the hERG deficiency in diabetic cardiomyopathy.

In our study, a reduction in the expression of the hERG protein and hERG current was observed after treatment with high glucose (Fig. 1); nevertheless, hERG channel kinetics were not altered (Fig. 2). These results indicated that high glucose inhibited the hERG channel through a disruption in hERG protein expression. It is well known that hERG protein trafficking is regulated through a cytosolic chaperone network [13]. Currently, only a few chaperones participating in hERG trafficking have been confirmed, including Hsp70, Hsp90, GM130 and the 14-3-3 protein [19]. Among these chaperones, Hsp70 and Hsp90 are the best characterized and play the most important roles. In the ER, Hsp70 binds to the initial hERG, while in the cytoplasm, Hsp90 binds to the terminal hERG to assist in folding [20]. These proteins co-precipitate with the hERG protein and are essential for hERG maturation [20, 21]. Therefore, we determined whether high glucose levels modified the expression levels of Hsp90 and/or Hsp70 and the association of hERG channels with Hsp90 and/or Hsp70. In our study, we found that the expression of Hsp90 was reduced but Hsp70 expression was not altered in the presence of high glucose (Fig. 3A and B). These results are similar to those of a previous study showing that Hsp90 expression was significantly decreased in diabetic glomeruli [22]. Furthermore, treatment with high glucose at $75 \mathrm{mM}$ reduced the formation of hERG/Hsp90 complexes, while the formation of hERG/Hsp70 complexes was increased (Fig. $3 \mathrm{C}$ and D). Taken together, these data demonstrate that the high-glucose-induced trafficking 
deficiency is likely caused by the inhibition of Hsp90 expression and alterations in hERG/ Hsp90 interactions.

A trafficking deficiency in the hERG channel is activated through the ER stress response [23-25]. The UPR, which indicates the occurrence of an ER stress response, increases the synthesis of chaperone proteins and prevents the accumulation of unfolded protein in the ER $[26,27]$. The UPR comprises three pathways: ATF-6, IRE- 1 and PERK, and ATF-6 is a key regulator in the initial response [28]. ATF- 6 comprises two forms: an inactive form ( $90 \mathrm{kDa}$ ) and an active, cleaved form (50 kDa). Cleaved ATF- 6 initiates the expression of target genes and increases the expression of chaperone proteins to assist in hERG channel folding [26, 28]. Calnexin and calreticulin are two key ER chaperone proteins that ensure the proper folding of newly synthesized proteins and other QC mechanisms [27, 29]. The results of our study revealed that the application of high glucose impedes hERG channel trafficking. We consistently found that a decreased expression of inactive ATF- 6 and increased expression of active, cleaved ATF- 6 were induced in the presence of high glucose (Fig. 4A). Moreover, the expression of calnexin was up-regulated, although calreticulin expression was not changed (Fig. 4B and C). These results indicate that the trafficking deficiency in the hERG channel caused by high glucose activates the UPR. In addition, recent studies have shown that misfolded and retained trafficking-defective proteins are eventually degraded by a process termed ER-associated degradation [24, 30]. Thus, we used western blot analysis to monitor the rate of hERG degradation. The results indicated that the application of high glucose for $48 \mathrm{~h}$ enhances the degradation rate of mature hERG channels (Fig. 5).

Several studies have reported that hERG depression is induced by oxidative stress, and indeed insulin has antioxidant properties and reduces intracellular levels of ROS [31-33]. Moreover, insulin improves $\mathrm{I}_{\mathrm{Kr}} / \mathrm{hERG}$ function in IDDM rabbits via its antioxidative actions [9]. Another study demonstrated that insulin improves depressed $I_{\text {to }}$ in diabetic rats [34]. In the present study, insulin significantly increased the expression of the hERG protein and hERG current (Fig. 6A and B). Furthermore, the high-glucose-induced reduction of the hERG protein and hERG current can be ameliorated by insulin, due to insulin's effect in promoting the expression of the hERG channel (Fig. 6C to E). The reduced expression level of Hsp90 negatively affects hERG channel folding and maturation [20]. The expression of Hsp90 thus plays an important role in the expression of the hERG channel. Therefore, in this study, we assessed the effect of insulin and high-glucose on Hsp90 protein expression. The results (Fig. 6F) revealed that, in the presence of insulin, the depression of Hsp90 that was caused by high glucose was significantly recovered, and the expression of Hsp90 was increased. These results indicated that insulin has the potential to improve the hERG protein trafficking deficiency, demonstrating an important mechanism whereby insulin ameliorates high-glucose-induced hERG inhibition. Our results demonstrate that high-glucose-induced hERG dysfunction is both preventable and reversible, and insulin is highly effective in the treatment of these diabetic electrical problems.

In summary, our results provide the first evidence that high glucose induces hERG channel deficiency by impairing hERG channel trafficking. This finding illustrates a novel intracellular mechanism underlying the diabetic-induced hERG channel expression deficiency. Furthermore, we demonstrated that insulin is highly effective in rescuing the hERG deficiency induced by high-glucose treatment in hERG-HEK cells. Taken together, these findings will greatly improve the current understanding of the hERG deficiency in diabetic cardiomyopathy and may provide an effective therapeutic approach for the electrical problems in diabetic patients.

\section{Acknowledgements}

This work was financially supported through grants from the National Natural Science Foundation of China (No.31173050), the Research Fund for the Doctoral Program of Higher Education of China (No. 20122307110007), Funds for Creative Research Groups of the 


\begin{tabular}{|c|c|c|}
\hline Cellular Physiology & Cell Physiol Biochem 2015;37:284-296 & \\
\hline and Biochemistry & $\begin{array}{l}\text { DOI: 10.1159/000430353 } \\
\text { Published online: August 24, } 2015\end{array}$ & $\begin{array}{l}\text { O } 2015 \text { S. Karger AG, Basel } \\
\text { www.karger.com/cpb }\end{array}$ \\
\hline
\end{tabular}

National Natural Science Foundation of China (81421063) and the Science Foundation of Heilongjiang Province for Youth (QC2015099).

\section{Disclosure Statement}

There are no conflicts of interest to declare.

\section{References}

1 Christensen PK, Gall MA, Major-Pedersen A, Sato A, Rossing P, Breum L, Pietersen A, Kastrup J, Parving HH: Qtc interval length and QT dispersion as predictors of mortality in patients with non-insulin-dependent diabetes. Scand J Clin Lab Invest 2000;60:323-332.

2 Okin PM, Devereux RB, Lee ET, Galloway JM, Howard BV, Strong Heart S: Electrocardiographic repolarization complexity and abnormality predict all-cause and cardiovascular mortality in diabetes: the strong heart study. Diabetes 2004;53:434-440.

3 Rossing P, Breum L, Major-Pedersen A, Sato A, Winding H, Pietersen A, Kastrup J, Parving HH: Prolonged QTc interval predicts mortality in patients with Type 1 diabetes mellitus. Diabet Med 2001;18:199-205.

4 Veglio M, Chinaglia A, Cavallo-Perin P: Qt interval, cardiovascular risk factors and risk of death in diabetes. J Endocrinol Invest 2004;27:175-181.

5 Wang DW, Kiyosue T, Shigematsu S, Arita M: Abnormalities of K(+) and $\mathrm{Ca}(2+)$ currents in ventricular myocytes from rats with chronic diabetes. Am J Physiol 1995;269:H1288-1296.

6 Zhang Y, Xiao J, Lin H, Luo X, Wang H, Bai Y, Wang J, Zhang H, Yang B, Wang Z: Ionic mechanisms underlying abnormal QT prolongation and the associated arrhythmias in diabetic rabbits: A role of rapid delayed rectifier K(+) current. Cell Physiol Biochem 2007;19:225-238.

7 Curran ME, Splawski I, Timothy KW, Vincent GM, Green ED, Keating MT: A molecular basis for cardiac arrhythmia: hERG mutations cause long QT syndrome. Cell 1995;80:795-803.

8 Keating MT, Sanguinetti MC: Molecular and cellular mechanisms of cardiac arrhythmias. Cell 2001;104:569-580.

9 Zhang Y, Xiao J, Wang H, Luo X, Wang J, Villeneuve LR, Zhang H, Bai Y, Yang B, Wang Z: Restoring depressed hERG K(+) channel function as a mechanism for insulin treatment of abnormal QT prolongation and associated arrhythmias in diabetic rabbits. Am J Physiol Heart Circ Physiol 2006;291:H1446-1455.

10 Shu L, Zhang W, Su G, Zhang J, Liu C, Xu J: Modulation of herg K(+) channels by chronic exposure to activators and inhibitors of PKA and PKC: Actions independent of PKA and PKC phosphorylation. Cell Physiol Biochem 2013;32:1830-1844.

11 Pakladok T, Almilaji A, Munoz C, Alesutan I, Lang F: Pikfyve sensitivity of hERG channels. Cell Physiol Biochem 2013;31:785-794.

12 Hebert DN, Molinari M: In and out of the ER: Protein folding, quality control, degradation, and related human diseases. Physiol Rev 2007;87:1377-1408.

13 Vandenberg JI, Perry MD, Perrin MJ, Mann SA, Ke Y, Hill AP: hERG K(+) channels: structure, function, and clinical significance. Physiol Rev 2012;92:1393-1478.

14 Mehnert JM, Brandenburger M, Grunow B: Electrophysiological characterization of spontaneously contracting cell aggregates obtained from rainbow trout larvae with multielectrode arrays. Cell Physiol Biochem 2013;32:1374-1385.

15 Shi H, Liu KJ: Effects of glucose concentration on redox status in rat primary cortical neurons under hypoxia. Neurosci Lett 2006;410:57-61.

16 Gu DF, Li XL, Qi ZP, Shi SS, Hu MQ Liu DM, She CB, Lv YJ, Li BX, Yang BF: Blockade of hERG K+ channel by isoquinoline alkaloid neferine in the stable transfected HEK293 cells. Naunyn Schmiedebergs Arch Pharmacol 2009;380:143-151.

17 Joshi M, Kotha SR, Malireddy S, Selvaraju V, Satoskar AR, Palesty A, McFadden DW, Parinandi NL, Maulik N: Conundrum of pathogenesis of diabetic cardiomyopathy: Role of vascular endothelial dysfunction, reactive oxygen species, and mitochondria. Mol Cell Biochem 2014;386:233-249. 


\section{Cellular Physiology Cell Physiol Biochem 2015;37:284-296

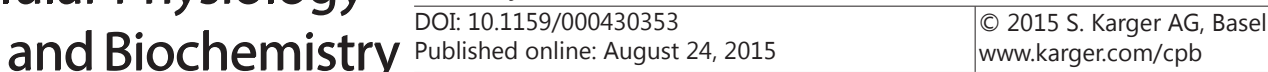 \\ Shi et al.: High Glucose Inhibits hERG Trafficking}

18 Guo J, Li X, Shallow H, Xu J, Yang T, Massaeli H, Li W, Sun T, Pierce GN, Zhang S: Involvement of caveolin in probucol-induced reduction in hERG plasma-membrane expression. Mol Pharmacol 2011;79:806-813.

19 Dennis A, Wang L, Wan X, Ficker E: hERG channel trafficking: Novel targets in drug-induced long QT syndrome. Biochem Soc Trans 2007;35:1060-1063.

20 Ficker E, Dennis AT, Wang L, Brown AM: Role of the cytosolic chaperones Hsp70 and Hsp90 in maturation of the cardiac potassium channel herg. Circ Res 2003;92:e87-100.

21 Delisle BP, Anson BD, Rajamani S, January CT: Biology of cardiac arrhythmias: Ion channel protein trafficking. Circ Res 2004;94:1418-1428.

22 Kim YS, Sohn E, Jung DH, Lee YM, Kim CS, Kim J, Kim JS: Expression of heat shock protein 90 in the kidneys of diabetic db/db mice. Eur Rev Med Pharmacol Sci 2014;18:2198-2204.

23 Smith JL, McBride CM, Nataraj PS, Bartos DC, January CT, Delisle BP: Trafficking-deficient hERG $\mathrm{K}(+)$ channels linked to long qt syndrome are regulated by a microtubule-dependent quality control compartment in the ER. Am J Physiol Cell Physiol 2011;301:C75-85.

24 Wang Y, Huang X, Zhou J, Yang X, Li D, Mao H, Sun HH, Liu N, Lian J: Trafficking-deficient G572R-hERG and E637K-hERG activate stress and clearance pathways in endoplasmic reticulum. PloS one 2012;7:e29885.

25 Wu ZY, Yu DJ, Soong TW, Dawe GS, Bian JS: Progesterone impairs human ether-a-go-go-related gene (hERG) trafficking by disruption of intracellular cholesterol homeostasis. J Biol Chem 2011;286:22186-22194.

26 Malhotra JD, Kaufman RJ: The endoplasmic reticulum and the unfolded protein response. Semin Cell Dev Biol 2007;18:716-731.

27 Paschen W: Endoplasmic reticulum: A primary target in various acute disorders and degenerative diseases of the brain. Cell calcium 2003;34:365-383.

28 Lynch JM, Maillet M, Vanhoutte D, Schloemer A, Sargent MA, Blair NS, Lynch KA, Okada T, Aronow BJ, Osinska H, Prywes R, Lorenz JN, Mori K, Lawler J, Robbins J, Molkentin JD: A thrombospondin-dependent pathway for a protective ER stress response. Cell 2012;149:1257-1268.

29 Yoshida H, Okada T, Haze K, Yanagi H, Yura T, Negishi M, Mori K: ATF6 activated by proteolysis binds in the presence of NF-Y (CBF) directly to the cis-acting element responsible for the mammalian unfolded protein response. Mol Cell Biol 2000;20:6755-6767.

30 Zhang K, Zhi D, Huang T, Gong Y, Yan M, Liu C, Wei T, Dong Z, Li B, Yang B: Berberine induces herg channel deficiency through trafficking inhibition. Cell Physiol Biochem 2014;34:691-702.

31 Aikawa R, Nawano M, Gu Y, Katagiri H, Asano T, Zhu W, Nagai R, Komuro I: Insulin prevents cardiomyocytes from oxidative stress-induced apoptosis through activation of PI3 kinase/Akt. Circulation 2000;102:28732879.

32 Kang S, Song J, Kang H, Kim S, Lee Y, Park D: Insulin can block apoptosis by decreasing oxidative stress via phosphatidylinositol 3-kinase- and extracellular signal-regulated protein kinase-dependent signaling pathways in HepG2 cells. Eur J Endocrinol 2003;148:147-155.

33 Wohaieb SA, Godin DV: Alterations in free radical tissue-defense mechanisms in streptozocin-induced diabetes in rat. Effects of insulin treatment. Diabetes 1987;36:1014-1018.

34 Xu Z, Patel KP, Lou MF, Rozanski GJ: Up-regulation of K(+) channels in diabetic rat ventricular myocytes by insulin and glutathione. Cardiovasc Res 2002;53:80-88. 